\title{
Auralization of Hybrid Wing Body Aircraft Flyover Noise from System Noise Predictions
}

\author{
Stephen A. Rizzi ${ }^{1}$ \\ NASA Langley Research Center, Hampton, VA 23681, USA \\ Aric R. Aumann ${ }^{2}$ \\ Analytical Services and Materials, Inc., Hampton, VA 23666, USA \\ and \\ Leonard V. Lopes ${ }^{3}$ and Casey L. Burley ${ }^{4}$ \\ NASA Langley Research Center, Hampton, VA 23681, USA
}

\begin{abstract}
System noise assessments of a state-of-the-art reference aircraft (similar to a Boeing 777200ER with GE90-like turbofan engines) and several hybrid wing body (HWB) aircraft configurations were recently performed using NASA engine and aircraft system analysis tools. The HWB aircraft were sized to an equivalent mission as the reference aircraft and assessments were performed using measurements of airframe shielding from a series of propulsion airframe aeroacoustic experiments. The focus of this work is to auralize flyover noise from the reference aircraft and the best HWB configuration using source noise predictions and shielding data based largely on the earlier assessments. For each aircraft, three flyover conditions are auralized. These correspond to approach, sideline, and cutback operating states, but flown in straight and level flight trajectories. The auralizations are performed using synthesis and simulation tools developed at NASA. Audio and visual presentations are provided to allow the reader to experience the flyover from the perspective of a listener in the simulated environment.
\end{abstract}

$\begin{array}{ll} & \\ a_{k}, f_{k}, \phi_{k} & =\text { Nomplitude, frequency and phase of } k^{\text {th }} \text { tone } \\ B P F & =\text { blade passage frequency } \\ E P N L & =\text { effective perceived noise level, EPNdB } \\ H W B & =\text { hybrid wing body } \\ L F O & =\text { low frequency oscillation } \\ M & =\text { Mach number } \\ P A A & =\text { propulsion airframe aeroacoustics } \\ P N L T & =\text { tone-corrected perceived noise level, PNdB } \\ R-S & =\text { rotor-stator (interaction tone noise) } \\ S_{k}(t) & =\text { pressure time history of } k^{\text {th }} \text { tone, Pa } \\ S E L & =\text { sound exposure level, dB } \\ S P L & =\text { sound pressure level, dB } \\ S P L_{A} & =\text { A-weighted sound pressure level, dBA } \\ \theta & =\text { polar directivity angle, degrees (jet exhaust axis at } 180 \text { degrees) } \\ \psi & =\text { azimuthal directivity angle, degrees }\end{array}$

\footnotetext{
${ }^{1}$ Senior Research Engineer, Structural Acoustics Branch, MS 463, Associate Fellow AIAA

2 Software Engineer, Structural Acoustics Branch, MS 463

${ }^{3}$ Research Engineer, Aeroacoustics Branch, MS 461, Member AIAA

${ }^{4}$ Senior Research Engineer, Aeroacoustics Branch, MA 461, Senior Member AIAA
} 


\section{Introduction}

A LTHOUGH aircraft noise has been significantly reduced since the introduction of commercial, jet-powered aircraft over fifty years ago, this has been largely due to reductions in source noise through the development of high bypass turbofan engines and improved engine performance. These improvements have enabled twin engine designs that have significantly lower noise emissions than earlier four engine designs. Notwithstanding these gains, aircraft noise continues to be a concern due to continued growth in air traffic, increasingly stringent environmental goals, and operational limitations imposed by airport authorities. Hence, new designs for even quieter aircraft are being considered. ${ }^{1,2}$ In particular, the goal of the NASA Environmentally Responsible Aviation (ERA) project is a cumulative effective perceived noise level (EPNL) of 42 EPNdB below the Federal Aviation Administration's (FAA) Stage 4 noise certification level, with simultaneous reductions in fuel burn and NOx. ${ }^{3}$ If implemented, this goal represents about a $27-30 \mathrm{EPNdB}$ cumulative reduction compared to today's best aircraft, an amount comparable to the reduction achieved over the last thirty years.

One of the concepts under consideration in the ERA project is the hybrid wing body (HWB). The HWB configuration represents an unconventional aircraft concept that introduces the fundamental change of installing the engines on top of a lifting body airframe and eliminating traditional high-lift systems with multi-element flaps. In the past, full system noise studies of the HWB have proven to be a challenge due to the inability to analyze advanced configurations, in particular in characterizing the aeroacoustic effects related to the interaction of the propulsion system with the airframe, called propulsion airframe aeroacoustics (PAA). However, a recent large scale PAA experiment was performed, from which data characterizing the effect of airframe shielding were measured. ${ }^{4}$ System noise assessments of several HWB aircraft were made by combining flight path, aircraft sizing, and engine sizing analyses with Aircraft Noise Prediction Program ${ }^{5}$ (ANOPP) system noise analyses utilizing that shielding measurement. ${ }^{6}$ While all variations were found to exhibit noise reductions (relative to Stage 4) greater than the reference state-of-the art (SOA) aircraft, the best achieved a 42.9 EPNdB cumulative noise reduction, exceeding the ERA noise goal. Further, contours of the ground sound exposure levels (SEL) indicated a nearly $80 \%$ reduction in the $85 \mathrm{~dB}$ SEL approach and takeoff contour area relative to the SOA aircraft. While quantitative, these integrated metrics provide no sense of the sound characteristics as heard by a listener on the ground. The ability to auralize, i.e., render to audible sound, a simulated flyover has multiple benefits. It provides another means of expressing the societal benefit of low noise concepts to stakeholders in a form that is inherently understandable. It also provides a feedback mechanism to the technologist developing noise reduction concepts.

Concurrent with improvements to its system noise prediction tools, NASA has developed the capability to auralize aircraft flyover noise through a set of tools for source noise synthesis and flyover simulation. ${ }^{7-10}$ The tools take into account the source directivity, shielding effects, propagation effects including spreading loss, atmospheric absorption, Doppler, ground plane reflections, and listener effects which permit coupling to an interactive virtual environment.

Common to both the system noise prediction and the auralization are the source noise definitions, which are obtained through several ANOPP source noise modules. However, the method of propagation between the system noise prediction and auralization differs. In the system noise prediction, propagation of the source noise to a ground observer is performed on a 1/3-octave band basis. For auralization, propagation of the synthesized source pressure time histories occurs in the time domain. Common features and differences of each formulation are discussed, as well as workarounds for capabilities that exist in one approach and not the other.

This paper develops auralization methods suitable for use with system noise prediction tools and applies those to two aircraft configurations similar to the reference SOA aircraft and the best HWB configuration (designated C11) considered in the prior assessment study. ${ }^{6}$ The focus of this effort is not to auralize the certification profiles considered in the prior assessments, but to establish a validated approach for utilizing system noise predictions tools for auralization. To that end, source directivity data from ANOPP serve as input into the source noise synthesis. The data are largely, but not entirely, consistent with that used in prior assessment analyses. Propagation of the resulting pressure time history to observer locations is performed in a manner consistent with the earlier simulation work. Integrated metrics obtained using the simulation approach are compared with those obtained from ANOPP under the same conditions to validate the methodology. Differences between the two approaches are explored through consideration of system and component level metrics. Finally, auralizations combined with visualization are provided to allow one to experience the flyover from the perspective of a listener in the simulated outdoor environment. 


\section{System Noise Prediction}

\section{A. Aircraft and Engine System Models}

The reference SOA and HWB aircraft configurations considered in the prior assessments ${ }^{6}$ served as starting points for the current effort. In brief, the SOA aircraft was based upon a NASA model of the Boeing 777-200ER tube-and-wing aircraft with GE90-like engines. The HWB C11 aircraft had an equivalent mission definition as the SOA aircraft and utilized the same engines, but these were scaled to achieve a minimum gross weight while retaining comparable take-off and climb performance. A summary of aircraft system data is shown in Table 1 for the SOA and NASA HWB C11 aircraft. An artist depiction of the NASA HWB C11 configuration is shown in Figure 1 to familiarize the reader with the vehicle concept. Note however that the depiction shown indicates verticals at the wingtips instead of at the inboard locations specified for the $\mathrm{C} 11$ configuration.

Table 1: Summary of the aircraft system data.

\begin{tabular}{|l|r|r|}
\hline & \multicolumn{1}{|c|}{$\begin{array}{c}\text { 777-like } \\
\text { SOA }\end{array}$} & \multicolumn{1}{c|}{$\begin{array}{c}\text { NASA } \\
\text { HWB C11 }\end{array}$} \\
\hline Weight-takeoff (lbs) & 656,000 & 590,436 \\
\hline Weight-landing (lbs) & 459,200 & 413,305 \\
\hline Max Fuel (lbs) & 284,279 & 227,081 \\
\hline Engine SFC (lbm/hr/lbf) & 0.557 & 0.549 \\
\hline L/D (start of cruise) & 19.5 & 23.0 \\
\hline Thrust per Engine (static sea level) & 86,783 & 81,298 \\
\hline Throttle: Approach (full throttle =1.0) & 0.190 & 0.129 \\
\hline Throttle: Sideline & 1.0 & 1.0 \\
\hline Throttle: Cutback & 0.78 & 0.76 \\
\hline Takeoff Field-Length (ft) & 8648 & 8633 \\
\hline
\end{tabular}

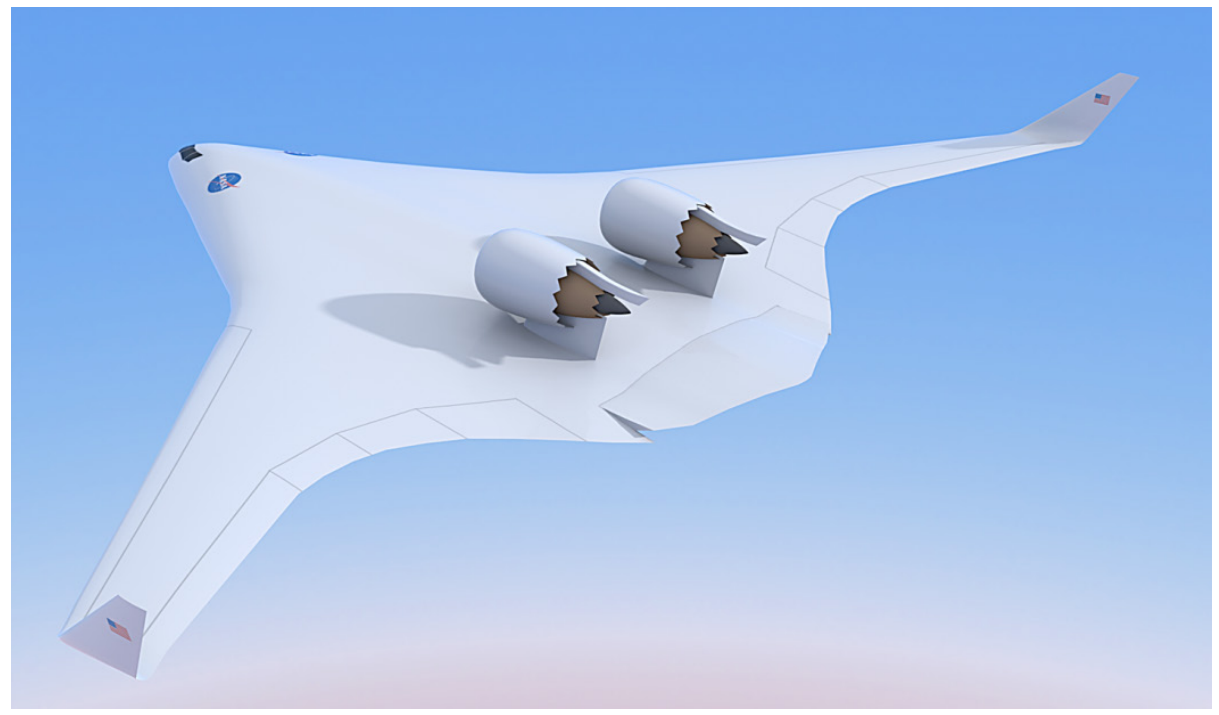

Figure 1: Artist depiction of a modified NASA HWB C11 [from Thomas et. al. ${ }^{6}$ ]

Some modifications to the reference SOA and HWB C11 aircraft models were made in the present investigation that were inconsistent with configurations used in the prior assessments. These included changes to the aircraft trajectory, ANOPP source modules, and ANOPP propagation modules. Some of these changes were required due to differences between the system noise prediction and the auralization methodologies. Others were discretionary to help better understand the simulation space. These changes are discussed in detail in subsequent sections of the paper. The net result is that the auralizations presented in this paper, while validated, do not represent the conditions and configurations considered in the prior assessments. Therefore, no attempt is made by the authors, nor should be made by the readers, to compare the two sets of results. 


\section{B. Source Noise Prediction}

The ANOPP modules used to generate component source noise directivities used in the auralization are listed in Table 2. These are the same as those considered by Thomas et $\mathrm{al},{ }^{6}$ except as noted below. Each isolated noise component may undergo some form of noise treatment. The word "treatment" here is a generic term to indicate any modifier of the isolated source, whether that be due to application of a liner, shielding or noise reduction technology.

The engine noise sources considered were the forward radiated fan noise, aft radiated fan noise, core noise, and jet noise. Forward radiated fan noise was comprised of broadband, rotor-stator (R-S) interaction tone, and combination tone sources. Aft radiated fan noise was comprised of broadband and R-S interaction tone sources. Note that combination tone noise, also referred to as multiple pure tone or buzzsaw noise, was included in this work but not in the prior assessment study. This discretionary change was made to highlight special synthesis considerations associated with this type of noise source. For both aircraft, fan noise attenuation from acoustic liners was incorporated using the forward and aft GE methods in the ANOPP TREAT module. ${ }^{11}$

The ANOPP engine noise predictions were calibrated for the SOA aircraft utilizing publicly available GE90 EPNL values, as described by Thomas el al. ${ }^{6}$ Because the SOA configuration has engines mounted under the wings, the calibration process used the ANOPP WING module to account for reflections of the aft radiated sources off the lower wing surface. Note however that the modeling method used in the WING module is not compatible with the auralization methodology. Therefore, for auralization purposes only, alternative means of modeling the effect of the wing reflection were employed, as discussed in section II.C The engine calibration factors themselves were unchanged from those used in the previous assessments. ${ }^{6}$

The calibrated GE90-like engine was subsequently scaled ${ }^{6}$ and modified to account for installation effects on the HWB aircraft. In particular, the effects of shielding are important for the HWB with its engines mounted above the wing body. Shielding was accounted for by adjusting the source level and directivity through the application of suppression to the predicted source noise. A suppression table was generated by calculating a suppression factor for each 1/3-octave band center frequency at every polar, $\theta$, and azimuthal, $\psi$, angle on the noise source hemisphere. The suppression tables were determined from experimental data ${ }^{4}$ and were provided as input to ANOPP and to the auralization. Forward and aft radiated suppression tables obtained from a broadband point source were applied to the core and all fan noise components, as indicated in Table 2. It is recognized, however, that the suppression of tonal noise may differ. ${ }^{6}$ A different suppression table was applied for jet noise to account for the distributed nature of the source. As in the previous study, the aft radiated fan noise was additionally attenuated by $5 \mathrm{~dB}$ to account for the effect of advanced liner technology and additional liner application area due to the crown pylon. As noted by Czech et $\mathrm{al}^{4}{ }^{4}$ an additional $0.25 \mathrm{~dB}$ was added to the noise of the chevron nozzle to account for the fact that the area of the chevron nozzle was $4 \%$ lower than the baseline.

Airframe noise sources for the SOA aircraft included the main and nose landing gear, leading edge slats, trailing edge noise, and trailing edge flaps. The HWB aircraft does not have flaps and, therefore, HWB airframe noise only included the main and nose landing gear, leading edge slat, and trailing edge noise. The landing gear and flap methods have been well validated and have shown excellent comparison with full-scale 777 data. ${ }^{12,13}$ The landing gear description for both the SOA and HWB aircraft was taken as that reported by Guo ${ }^{12}$ for the 777 . For the C11 configuration, nose and main gear noise were additionally suppressed to account for projected reductions associated with quiet landing gear technology.

\section{Auralization considerations}

For each operating condition investigated, the ANOPP source modules together with the treatments indicated in Table 2 were used to obtain noise source hemispheres with polar directivity angles ranging from $10^{\circ}$ (forward) to $170^{\circ}$ (aft), and azimuthal directivity angles ranging from $-90^{\circ}$ (port-side) to $90^{\circ}$ (starboard side), both in $10^{\circ}$ increments. Since the system noise prediction used herein was originally intended to calculate Federal Aviation Regulation (FAR), Part 36 certification metrics, source noise directivity data was exported from ANOPP over a 1/3octave band frequency range from $50 \mathrm{~Hz}-10 \mathrm{kHz}$. For general auralization purposes, this frequency range may be insufficient if perceptually important frequency content exists outside that bandwidth, e.g. below $50 \mathrm{~Hz}$. Both ANOPP and the auralization process interpolate the source noise directivity to obtain spectra at intermediate emission angles. If the hemisphere discretization is too coarse, essential features of the auralized sound may be missed, e.g. those associated with a highly directional fan tone. These are less likely to affect an integrated metrics calculation for the full system. Finally, auralization of arbitrary trajectories, e.g. a turning bank, would require source spectra on the upper hemisphere, something that is not generally considered in certification metrics calculations. 
Table 2: ANOPP prediction modules used to generate component source noise directivities and synthesis method used to generate pressure time histories. A check mark indicates a particular source was included.

\begin{tabular}{|c|c|c|c|c|}
\hline Noise Component & $\begin{array}{l}\text { ANOPP } \\
\text { Module }\end{array}$ & $\begin{array}{l}\text { SOA Aircraft } \\
\text { (Treatment) }\end{array}$ & $\begin{array}{c}\text { HWB C11 Aircraft } \\
\text { (Treatment) }\end{array}$ & $\begin{array}{c}\text { Synthesis Method } \\
\text { (Modifier) }\end{array}$ \\
\hline Fan Noise & \multirow{6}{*}{$\mathrm{BFN}^{14}$} & & & \\
\hline - Fwd broadband & & $\checkmark\left(\right.$ TREAT $\left.^{\dagger}\right)$ & $\checkmark\left(\right.$ TREAT $^{\dagger} /$ Suppress $\left.^{\natural}\right)$ & Broadband \\
\hline - Fwd R-S interaction tones & & $\checkmark\left(\right.$ TREAT $\left.^{\dagger}\right)$ & $\checkmark\left(\right.$ TREAT $^{\dagger} /$ Suppress $\left.^{\emptyset}\right)$ & Tonal $\left(\mathrm{LFO}^{10}\right)$ \\
\hline - Fwd combination tones & & $\checkmark\left(\right.$ TREAT $\left.^{\dagger}\right)$ & $\checkmark\left(\right.$ TREAT $^{\dagger} /$ Suppress $\left.^{\natural}\right)$ & Tonal $\left(\mathrm{LFO}^{10}\right)$ \\
\hline - Aft broadband & & $\begin{array}{c}\checkmark \\
\left(\mathrm{TREAT}^{\ddagger} / \mathrm{WING}^{\S}\right) \\
\end{array}$ & $\begin{array}{c}\checkmark \text { (TREAT }^{*} / \text { Suppress }^{\#} / \\
\left.\text { Adv. Liner }{ }^{*}\right)\end{array}$ & Broadband \\
\hline - Aft R-S interaction tones & & $\begin{array}{c}\checkmark \\
\left(\mathrm{TREAT}^{\ddagger} / \mathrm{WING}^{\S}\right)\end{array}$ & $\begin{array}{c}\checkmark \text { (TREAT }^{*} / \text { Suppress }^{\#} / \\
\left.\text { Adv. Liner }{ }^{*}\right)\end{array}$ & Tonal $\left(\mathrm{LFO}^{10}\right)$ \\
\hline Core Noise & $\mathrm{GECOR}^{15}$ & $\checkmark\left(\mathrm{WING}^{\S}\right)$ & $\checkmark\left(\right.$ Suppress $\left.^{\#}\right)$ & Broadband \\
\hline Jet Noise & ST2JET $^{16}$ & $\checkmark\left(\mathrm{WING}^{\S}\right)$ & $\checkmark$ (Suppress $^{\dagger \dagger} /$ Chev $\left.^{\| \uparrow \mid}\right)$ & Broadband $\left(\mathrm{LFO}^{7}\right)$ \\
\hline Landing Gear (approach only) & \multirow{3}{*}{$\mathrm{BAF}^{12}$} & & & \\
\hline - Nose & & $\checkmark$ & $\checkmark\left(\right.$ Adv. Gear $\left.{ }^{\dagger}\right)$ & Broadband \\
\hline - Main & & $\checkmark$ & $\checkmark\left(\right.$ Adv. Gear $\left.{ }^{\S \S}\right)$ & Broadband \\
\hline Flaps & $\mathrm{BAF}^{13}$ & $\bar{\checkmark}$ & $\mathrm{n} / \mathrm{a}$ & Broadband \\
\hline Slats & $\mathrm{BAF}^{17}$ & $\checkmark$ & $\checkmark$ & Broadband \\
\hline Trailing Edge & FNKAFM $^{18,19}$ & $\checkmark$ & $\checkmark$ & Broadband \\
\hline
\end{tabular}

Conventional liner

$\dagger$ Fan liner treatment (ANOPP TREAT module: GE forward method)

* Fan liner treatment (ANOPP TREAT module: GE aft method)

Shielding

$\S$ Reflection off lower wing surface (ANOPP WING module). Note: effective delta dB from applied for auralization.

- Experimental suppression in forward direction from broadband point source (from Czech et $\mathrm{al}^{4}$ )

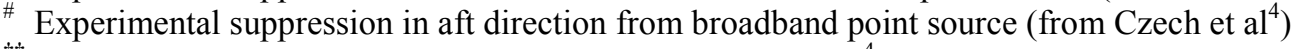

${ }^{\dagger}$ Experimental suppression from jet source (from Czech et $\mathrm{al}^{4}$ )

Noise reduction technology

\$Suppression for nose gear (from Berton et $\mathrm{al}^{20}$ )

$\S \S$ Suppression for main gear (from Berton et $\mathrm{al}^{20}$ )

9ी Suppression for advanced chevrons $(+0.25 \mathrm{~dB}$ penalty to account for $4 \%$ lower nozzle area than baseline)

* Advanced liner technology and added liner application are due to crown pylon $(-5 \mathrm{~dB})$ 
Another consideration for auralization has to do with the synthesis method employed. Depending on the nature of the source, either broadband or tonal synthesis methods were used, as indicated in Table 2. The details of the synthesis will be discussed in section III. For the present discussion, it is sufficient to note that broadband synthesis requires $1 / 3$-octave band data as input, while tonal synthesis requires tonal frequencies and amplitudes as input. Within ANOPP, the source hemispheres have any required treatment applied before propagation. The propagation module requires all source hemispheres to be specified in 1/3-octave bands. For auralization of broadband sources, ANOPP writes out the 1/3-octave band source hemispheres post-treatment and pre-propagation. The analogous operation is insufficient for auralization of tonal sources, in particular for forward and aft radiated fan R-S interaction tones, because the process of summing tones into 1/3-octave bands loses critical information for synthesis, namely the tonal frequencies and levels. Therefore, modifications to ANOPP were required to obtain fan tone frequencies and amplitudes from within the source module itself, that is, pre-treatment and pre-propagation. As such, treatment for tonal sources was performed as a separate processing operation outside of ANOPP prior to synthesis. This was strictly an implementation issue, but one that nevertheless had to be addressed for using the system noise prediction program ANOPP to generate source hemispheres for input to the synthesis.

Finally, because some ANOPP modules allow Doppler to be turned off while others do not, all ANOPP source hemispheres generated herein had Doppler turned on for consistency. The implications of this on the auralization process will be discussed in section III.

\section{Propagation}

Propagation in ANOPP is performed on source hemispheres expressed as $1 / 3$-octave band spectra and accounts for the spherical spreading loss, atmospheric absorption and time delay along a straight-line path between the moving source and a stationary observer. In this study, it includes the effect of ground plane reflection and, in the case of the SOA aircraft, the reflection of aft-radiated engine sources off the wing. Each of these effects is briefly described in order to later contrast it with the propagation approach used for auralization. Additional details may be found in the ANOPP manual. ${ }^{5}$

The straight-line path between the source and observer is computed at evenly spaced emission times. Starting at the time corresponding to the minimum distance between the source and observer, ANOPP marches forward and backward in time along the flight trajectory. The slant range, i.e. the straight line distance between the interpolated emission position and the observer, is used to compute the spherical spreading loss. Atmospheric absorption at 1/3octave band center frequencies is accumulated along the straight-line path through a stratified medium. An average speed of sound is calculated along the slant range and is used to determine the time delay. The spreading loss and absorption are applied to the source spectra, interpolated to each emission angle, to get the time-delayed spectra at the observer. These are subsequently interpolated at equally spaced receiver time increments to obtain a time history of $1 / 3$-octave band spectra at the observer.

When the ground plane reflection is considered, the reflected path incurs an additional time delay, spreading loss, and attenuation according to either a hard surface (infinite) or finite impedance boundary. The reflected path spectrum is added to the direct path spectrum to obtain the propagated spectrum at the observer.

Finally, for the SOA tube-and-wing configuration, there is potential for aft-radiated engine noise to be reflected from the lower wing surface in addition to the direct path. ANOPP provides a capability, through its WING module, to estimate this effect based on modeling the wing as a quadrilateral reflective surface. The reflected path, if any, is a function of this geometry and its relationship to the observer. Hence it is performed as part of the propagation processing. For calibration of the predicted engine noise, the core, jet and fan exit sources included this effect to predict the reflected noise as well as the direct noise at the certification points. However, there is no analogous process for auralization. Therefore, the effect of wing reflection for the SOA aircraft was achieved by performing ANOPP analyses with and without the WING module to determine a delta EPNdB at the certification points. A delta $\mathrm{dB}$ calibration factor was subsequently applied to the applicable source hemispheres such that the EPNdB computed without the WING module matched that computed with the WING module.

\section{Auralization Methodology}

Like the system noise prediction, the auralization methodology takes a source-path-receiver approach. Pressure time histories of the source are synthesized from 1/3-octave band or tonal amplitude source noise hemispheres obtained from ANOPP. Propagation of the pressure time histories to a ground observer is performed in the time domain based on the path, and simulates spherical spreading loss, atmospheric absorption, Doppler shift via timevarying propagation time delay, and ground plane reflection. The received pressure time history for the flyover may 
be post-processed to obtain integrated metrics, or auralized with or without an additional step of three-dimensional (3D) audio simulation.

\section{A. Source Noise Synthesis}

The pressure time history at the source position is synthesized at emission time based upon the instantaneous ANOPP source spectrum. The instantaneous source spectrum is a function of both the emission angle and the operational state of the aircraft. The emission angle is determined by the straight line path between the source and receiver and is typically calculated at an update rate on the order of $100 \mathrm{~Hz}$. The operational state is specified at waypoints in the trajectory at a much lower rate on the order of seconds. Note that because convective amplification is incorporated in the source noise hemispheres, its effect is automatically realized in the synthesized signal. This synthesis approach has been implemented in the NASA Aircraft Source Noise Generator (ASoNG) synthesis $\operatorname{program}^{7-10}$ and is depicted in Figure 2.

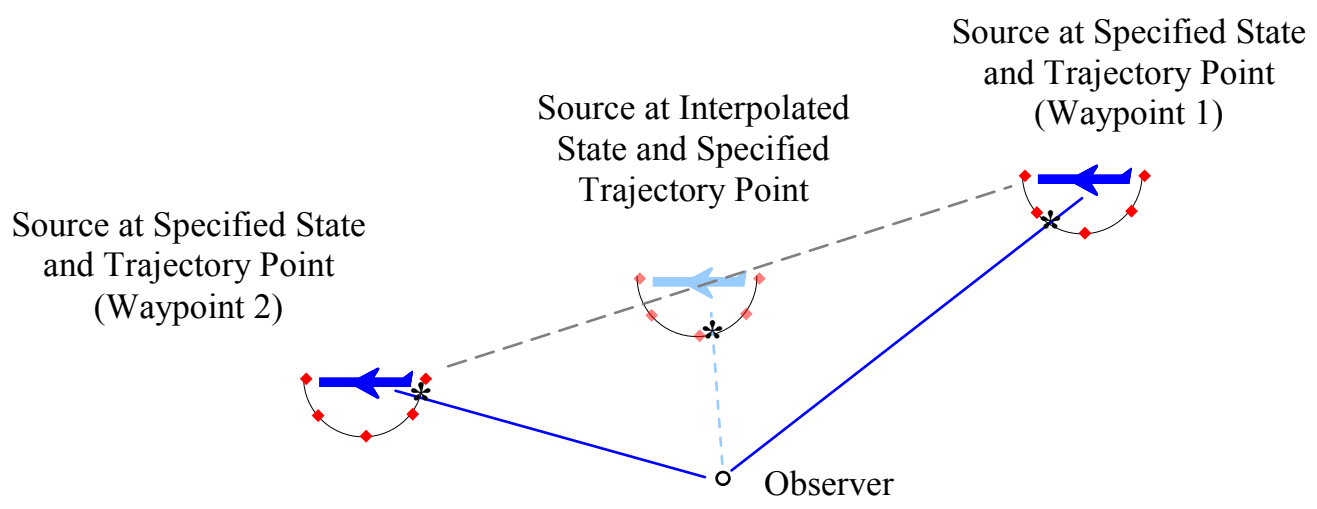

Figure 2: Synthesis is performed at the directivity angle (denoted by *) at the time of emission.

The ASoNG program synthesizes pressure time histories in a manner dependent on the source spectrum specified. A pressure time history that continually evolves with changes in source directivity is critical in order to avoid sudden changes in character resulting from a discretized source spectrum. For spectra expressed in $1 / 3$-octave band or narrowband form, ASoNG synthesizes the broadband noise using an overlap-add technique. ${ }^{7,8}$ For spectra expressed as tonal amplitudes, ASoNG synthesizes the noise in the time domain in a manner which permits changes in amplitude and frequency as a function of time. ${ }^{10}$ In the present work, ASoNG generates pressure time histories separately for each source noise component via the synthesis methods indicated in Table 2. The output of the synthesis process is analogous to the signal that a single microphone would record at some reference distance near the flying source. As the source moves, the emission angle changes in a continuous fashion and, by analogy, the microphone location smoothly traverses the hemisphere below the aircraft. In this manner, the source directivity is embedded in the synthesized signal and does not need to be explicitly represented in the propagation stage. This approach to synthesis makes simulation of arbitrary trajectories straightforward.

\section{Broadband Synthesis}

Broadband synthesis is performed as a subtractive synthesis operation using an overlap-add procedure. ${ }^{78}$ For each processing block, the instantaneous 1/3-octave band source spectrum is obtained via interpolation and converted to narrowband by evenly distributing the energy within each $1 / 3$-octave band across the appropriate number of evenly spaced narrow bins within the band. Since the source spectra lack phase, each narrowband component is assigned random phase. The resulting complex function acts as a filter of white noise through frequency domain convolution, and the result is inversely transformed to obtain the pressure time history. The next processing block corresponds to a point later in the trajectory. It is not contiguous, but overlaps the preceding block. Its processed output time history is added to that of the previous block at a time offset (hop size) corresponding to the amount of overlap. In this manner, the synthesized signal smoothly transitions with changes in source directivity.

Another implementation issue arising from the use of ANOPP for source hemispheres has to do with Doppler shift. The propagation phase of the auralization simulates Doppler, as described in section III.B. Therefore the Doppler shift included in the ANOPP source hemispheres must be removed prior to synthesis so that Doppler can 
then be simulated in the propagation phase. The action of removing the Doppler shift entails determining the upper and lower limits of each non-Doppler shifted band from the inverse Doppler relation

$$
f_{\text {non-Doppler }}^{i}=f_{\text {Doppler }}^{i}(1-M \cos \theta)
$$

where $f_{\text {non-Doppler }}^{i}$ are the upper and lower limits of the $i^{\text {th }}$ non-shifted 1/3-octave band, $f_{\text {Doppler }}^{i}$ are the upper and lower limits of the $i^{\text {th }}$ Doppler shifted 1/3-octave band, $M$ is the Mach number and $\theta$ is the emission angle. Note that in this case, the Doppler shifted bands are the standard $1 / 3$-octave bands $(50,63,80, \ldots \mathrm{Hz})$. The non-shifted limits are computed for each processing block because the Mach number and emission angle can change. Next the sound pressure level in each band is converted to narrowband by evenly distributing the energy reported at the standard 1/3-octave band center frequencies across the appropriate number of evenly spaced narrow frequency bins within the non-shifted bands. The remainder of the broadband synthesis process remains the same.

Finally, it is worth reiterating that the ANOPP source hemispheres for broadband sources are provided posttreatment. Therefore, syntheses based on these hemispheres reflect all applied treatments, calibration factors, suppressions, etc.

\section{Synthesis of Rotor-Stator Interaction Tones}

Synthesis of fan R-S interaction tones is performed in the time domain using an additive technique. The blade passage frequency (BPF) tone and its harmonics are represented as an amplitude and frequency-modulated cosine wave, as in

$$
s_{k}(t)=a_{k}(t) \cos \left(\phi_{k}(t)\right)
$$

where $a_{k}(t)$ is the amplitude envelope of the $k^{\text {th }}$ tone and $\phi_{k}(t)$ is the phase argument of the $k^{\text {th }}$ tone in radians. According to Eq. (2), each harmonic is characterized completely by two parameters; the amplitude and phase functions. The relationship of the time-variant frequency of the cosine term to the phase argument in the single-tone model is described by

$$
\phi_{k}(t)=2 \pi \int_{-\infty}^{t} f_{k}(\tau) d \tau+\phi_{o, k}
$$

where $f_{k}$ is the instantaneous frequency of the $k^{\text {th }}$ tone in $\mathrm{Hz}, \tau$ is a dummy variable of integration and $\phi_{o, k}$ is the initial phase. This expression for the phase allows for variations in frequency due to changes in operating condition or unsteadiness of the source, as described below. Note that a tone of constant (time-invariant) frequency $f$ will have a phase integral that becomes the familiar $2 \pi f t$ argument of a simple harmonic oscillator. The instantaneous frequency may be obtained from Eq. (3) by differentiating with respect to time as

$$
f_{k}(t)=\frac{1}{2 \pi} \frac{d\left(\phi_{k}(t)\right)}{d t} .
$$

The amplitudes of the BPF tone and its harmonics are varied continuously according to the instantaneous emission angle of the source noise hemisphere. The initial phase of each harmonic is randomized. Pressure time histories are synthesized for contiguous blocks of specified hop size. A continuous waveform is achieved by maintaining phase between subsequent blocks. Changes in the blade passage frequency do not occur within a single hemisphere corresponding to one operating condition, but may occur between different operating conditions, e.g. an engine spool up. All harmonics for each fan component (forward and aft-radiated) are summed to obtain the total $\mathrm{R}-\mathrm{S}$ interaction tonal noise for that component.

Because the BPF tone and its harmonics are known from ANOPP, there is no need to remove Doppler shift. However, since tonal hemispheres were written from within the BFN module, all treatments and calibration factors must be applied as part of the synthesis. As currently implemented, this operation is performed outside of ANOPP. Note that the treatment scaling factors are specified on a 1/3-octave band basis. The same scaling factor was applied to all tones within a band, not distributed amongst the tones within a band. This approach was found to be consistent with the ANOPP implementation.

\section{Synthesis of Combination Tones}

As previously indicated, synthesis of fan combination tones was considered in this work to highlight special considerations associated with this type of noise source. For both the SOA and HWB aircraft, its inclusion represents a departure from the prior assessment analyses. 
The processing chain for synthesis of combination tones differs from that used for synthesis of R-S interaction tones because of the manner in which the source noise prediction is made. Specifically, combination tone noise is predicted on a 1/3-octave band basis. However, combination tones are synthesized with the same additive technique as described above, so assumptions must be made about both the tonal amplitude and phase distribution.

First consider the non-Doppler shifted case. Combination tones occur at the shaft frequency $f_{k}$ and its harmonics

$$
f_{k}=k B P F / N B
$$

where $N B$ is the number of fan blades. Since no information is provided in the prediction to guide the distribution of energy within each $1 / 3$-octave band, it is equally distributed amongst the number of combination tones falling in each band. Further, each tone is assigned a random phase. Note however that additional processing is required for every $N B$-th combination tone, as it falls on a R-S BPF tone synthesized with some other phase. Summing the $N B$-th combination tone with the BPF tone can therefore unintentionally attenuate or amplify the resulting tone at this frequency. Instead, the $N B$-th combination tone is not synthesized at all, but its energy is equally distributed amongst all other combination tones falling in that $1 / 3$-octave band.

Next consider the Doppler shifted case. Data in this form is provided by the ANOPP source noise prediction. The amplitude distribution follows that of the non-Doppler shifted case, except that here the energy is equally distributed amongst the number of Doppler-shifted harmonics falling in each 1/3-octave band. The Doppler-shifted harmonics are determined by

$$
f_{k, \text { Doppler }}=f_{k} /(1-M \cos \theta)
$$

where $f_{k}$ are the non-Doppler shifted shaft frequencies determined from Eq. (5). Synthesis of combination tones, minus the BPF tone, occurs at non-Doppler shifted frequencies with the above amplitude distribution and random phase.

Another implementation issue can arise from the use of ANOPP for source hemispheres for combination tone noise. Consider a 22-blade fan with a BPF of $770 \mathrm{~Hz}$ in an overhead flyover (no Doppler) to demonstrate the issue. The shaft frequency is $35 \mathrm{~Hz}$. The mapping of shaft harmonics to the lower $1 / 3$-octave bands is shown in Table 3 . If the ANOPP prediction is made for the purpose of certification metrics calculations, the available data is in the range of $50 \mathrm{~Hz}-10 \mathrm{kHz}$. In this example, there is no ANOPP prediction available for the first shaft harmonic falling in the $31.5 \mathrm{~Hz}$ band $(<50 \mathrm{~Hz})$, so its synthesis amplitude is zero, i.e. that shaft harmonic is not synthesized. Further, for combination tone noise, the BFN source module predicts a nonzero level in every $1 / 3$-octave band, irrespective of whether or not a shaft harmonic falls in it. In this example, there are no shaft harmonics in the 50 and $80 \mathrm{~Hz}$ bands, but there are nonzero predicted levels in those bands. Since no tone is synthesized in those bands, the predicted energy is lost. While these differences are likely to have negligible effect on the total flyover noise, those

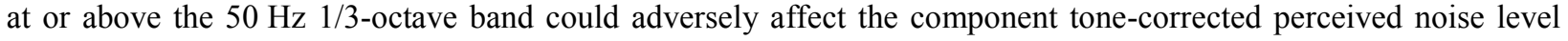
(PNLT), which penalizes large amplitude changes between 1/3-octave bands.

Table 3: Example distribution of shaft harmonics in lower 1/3-octave bands.

\begin{tabular}{ccccccccccc} 
1/3-OB center freq $(\mathrm{Hz})$ & 31.5 & 40 & 50 & 63 & 80 & 100 & 125 & 160 & 200 & 250 \\
\hline \# of shaft harmonics & 1 & 0 & 0 & 1 & 0 & 1 & 1 & 1 & 1 & 2
\end{tabular}

Finally, because the ANOPP source hemispheres for combination tone noise are reported in $1 / 3$-octave bands, they are provided post-treatment just like any other broadband source. Therefore, syntheses based on these hemispheres reflect all applied treatments and calibration factors.

\section{Temporal Variations}

The source noise hemispheres generated by ANOPP are determined from semi-empirical models. Therefore, at any emission angle, the source spectra are time invariant. Broadband and tonal syntheses based on these models faithfully reproduce the predicted spectra when averaged over time, but lack the temporal variations found in experimental data. The absence of temporal variations is observable and may diminish perceived realism. ${ }^{21}$

In an effort to increase the realism of synthesized noise, analyses of experimental data from static engine tests have been performed to characterize the fluctuations and introduce these into the synthesis process. To date, characterization of fluctuations in jet noise ${ }^{22}$ and tonal fan noise ${ }^{10}$ have been performed. The fluctuations in jet noise are described as low frequency $(<20 \mathrm{~Hz})$ oscillations (LFO) of each 1/3-octave band amplitude about the mean (predicted) value. Fluctuations in tonal fan noise are modeled as band-limited random fluctuations in amplitude and frequency with empirically derived cut-off frequencies $(<20 \mathrm{~Hz})$ and variances. 
The variation for jet noise is imparted on the synthesis by applying the analyzed time-varying delta $\mathrm{dB}$ on each $1 / 3$-octave band spectral amplitude after it has been interpolated for emission angle and operating state, but before being converted to narrowband. Time variations were imparted on tonal noise synthesis (see Eq. (2)) by casting each tonal amplitude and frequency in terms of its mean predicted value and a fluctuating component according to

$$
\begin{aligned}
& a_{k}(t)=a_{\text {mean }, k}+a_{\text {mod }, k}(t) \\
& f_{k}(t)=f_{\text {mean }, k}+f_{\text {mod }, k}(t)
\end{aligned}
$$

where $a_{\text {mean, } k}$ is the ANOPP predicted amplitude of the $k^{\text {th }}$ tone, $a_{\text {mod, } k}$ is the amplitude modulation of the $k^{\text {th }}$ tone around the mean value, $f_{\text {mean }, k}$ is the mean frequency (BPF or shaft frequency) of the $k^{\text {th }}$ tone, and $f_{\text {mod, } k}$ is the frequency modulation of the $k^{\text {th }}$ tone around the mean value. Using this approach, temporal variations were optionally applied to the component noise syntheses indicated in Table 2.

\section{B. Propagation}

Unlike ANOPP, propagation of the source noise to a ground observer occurs in the time domain through application of a time-dependent gain, time delay, and filter to the source noise. ${ }^{7,8}$ As in ANOPP, the propagation process accounts for spherical spreading loss, atmospheric absorption and time delay, as well as optionally including ground plane reflection. The time varying nature of these quantities is governed by the propagation path.

To maintain compatibility with the ANOPP propagation, a straight line path was assumed, although the capability for curved path simulation was recently added. ${ }^{23}$ The straight-line path between the source and observer is computed at evenly spaced emission times corresponding to the synthesis hop size. Spherical spreading loss is dependent on the slant range, giving a time-dependent negative gain. The time delay is a function of the speed of sound and slant range, and its time rate of change simulates Doppler shift. Because the time delay is not generally an integer multiple of the audio sampling rate (typically $44.1 \mathrm{kHz}$ ), fractional delay processing ${ }^{24}$ is required to avoid audible artifacts in the propagated sound. Note that there is an inconsistency in how the speed of sound is specified between ANOPP and the simulation. Recall that ANOPP computes an average speed of sound along the slant range. While the curved path simulation properly accounts for the speed of sound variation along the path, the straight-path simulation used herein only allows specification of a single speed of sound, regardless of the slant range. Consequently, the only accurate and consistent approach for a straight path is to specify a uniform atmosphere.

Atmospheric absorption is accumulated along the straight line path through the specified atmosphere at each 1/3octave band center frequency. The absorption curve is fit with a $2^{n}$-point spline and converted to a minimum phase finite impulse response (FIR) filter via an inverse fast Fourier transform (FFT), as described by Rizzi et al. ${ }^{7}$ The $^{-1}$ filter is slant range dependent and therefore varies in time with the moving source.

Once the time-dependent gain, time delay, and filter are known, the synthesized signal is propagated by filtering the time-delayed signal in the time domain and applying the time-dependent spreading loss to the result. The propagation stage is performed on a dedicated audio $\operatorname{server}^{25}$ as part of the NASA Community Noise Test Environment ${ }^{8}$ (CNoTE) simulator application. The output of the propagation stage is a pseudo-recording at the observer location.

Finally, ground plane reflection may be optionally applied according to either a hard surface (infinite) or finite impedance boundary. ${ }^{23,26}$ For a finite impedance boundary, the complex reflection coefficients are a function of frequency, angle of incidence, and flow resistivity of the ground plane and form an FIR filter when inverse transformed. Because the angle of incidence is dependent on the changing path, the applied filters are time varying. The effect of ground plane reflection is simulated with an image source. The reflected path is processed in a similar manner to that described above for the direct path, but with a time-varying delay line, gain, and atmospheric absorption filter associated with the image ray, as well as the time-varying ground plane filter. The interference caused by the addition of the propagated direct and reflected rays produces a comb filter effect, ${ }^{27}$ which alters the spectral content in a time-varying manner as the aircraft moves along its trajectory.

Pseudo-recordings of the propagated synthesized noise are post-processed using the ANOPP2 ${ }^{28}$ Acoustic Analysis API to generate PNLT and EPNL for comparison with ANOPP generated metrics. Because engineering units are maintained through the simulation process, the two sets of metrics will be shown to compare favorably.

\section{Receiver Simulation}

An optional processing step particular to auralization is the simulation of a $3 \mathrm{D}$ auditory environment at the listener position. If the sound is to be reproduced over headphones, binaural simulation ${ }^{29}$ may be used to make the propagated sound appear to come from the emission position. If the sound is to be reproduced over a speaker array, vector base amplitude panning ${ }^{30}$ may be used. Listener head tracking or scene tracking may be used to dynamically 
alter the soundscape. Receiver simulation with or without a corresponding 3D graphics simulation is performed as part of the CNoTE simulator application.

\section{Results}

Auralizations for the SOA and HWB C11 aircraft were performed for three straight and level trajectories with aircraft operating states that otherwise correspond to approach, sideline, and cutback operating conditions. Straight and level (S\&L) trajectories were chosen in lieu of certification flight trajectories to more easily compare the flyover noise of the two vehicles. On this basis alone, direct comparisons of EPNL levels and PNLT time histories cannot be made between the current and past studies. Each trajectory consisted of a $10,000 \mathrm{ft}$. segment $(-5000 \leq x \leq 5000)$ with centerline and sideline observers at $(0,0,4)$ and $(0,1476,4) \mathrm{ft}$., respectively. The flight vehicle altitude, speed, and angle of attack for each flight condition are listed in Table 4.

Table 4: Aircraft operating states used for auralization.

\begin{tabular}{|l|c|c|c|}
\cline { 2 - 4 } \multicolumn{1}{c|}{} & \multicolumn{3}{c|}{ Altitude (ft) AGL / Speed (knots) / Angle of Attack (deg) } \\
\cline { 2 - 4 } \multicolumn{1}{c|}{} & S\&L Approach & S\&L Sideline & S\&L Cutback \\
\hline SOA Aircraft & $394 / 126.5 / 9.15$ & $1000 / 180.4 / 6.84$ & $1492.8 / 177.2 / 6.84$ \\
\hline HWB C11 Aircraft & $394 / 115.4 / 12.81$ & $1000 / 156.2 / 10.28$ & $1492.8 / 156.2 / 9.95$ \\
\hline
\end{tabular}

As mentioned previously, a uniform atmosphere was specified to resolve inconsistencies between how the speed of sound is handled in the CNoTE auralization versus ANOPP. The following constants were specified: $15^{\circ} \mathrm{C}$ temperature, 1 atmosphere pressure, and $70 \%$ relative humidity. The atmospheric absorption in $\mathrm{dB} / \mathrm{m}$ was calculated based on the American National Standards Institute (ANSI) standard atmospheric absorption ${ }^{31}$ using the method described by Rizzi et al. ${ }^{7}$ From this, range-dependent 128-tap FIR atmospheric absorption filters were developed and were found to produce absorption consistent with the frequency-domain approach found in the ANOPP ATM module. Although both the CNoTE auralization and ANOPP can specify finite ground plane impedances, a hard surface was selected for expediency. A comparison of the two ground plane impedance implementations is left for further investigation.

The broadband synthesis used an FFT size of 8,192 points, with a hop size of 512 points, or $11.6 \mathrm{~ms}$ at a synthesis sampling rate of $44.1 \mathrm{kHz}$. Tonal synthesis was performed at the same sampling rate. All relevant components were summed in the time domain to generate the total aircraft flyover noise.

Pseudo-recordings were post-processed using the ANOPP $2^{28}$ Acoustic Analysis API with a block size of 32,768 and a segment length of $0.5 \mathrm{~s}$. Tone corrections located in bands below the $800 \mathrm{~Hz} 1 / 3$-octave band were omitted from the PNLT and EPNL calculations to minimize the influence of non-aircraft sources such as ground reflections. Finally, it was found that for long propagation distances, the pseudo-recording in the $10 \mathrm{kHz} 1 / 3$-octave band was effectively numerically zero. This resulted in anomalous tone penalties due to the manner in which PNLT is calculated for the highest 1/3-octave band. For the purposes of the PNLT and EPNL calculations only, the $10 \mathrm{kHz}$ $1 / 3$-octave band level was extrapolated from the $6.3 \mathrm{kHz}$ and $8 \mathrm{kHz}$ levels. This eliminated the artificially high tone penalty.

\section{A. Integrated Metrics}

The EPNL values calculated from the CNoTE auralization pressure time histories are provided in Table 5 for both aircraft under all three straight and level flight conditions. As expected, the HWB levels are lower than the SOA aircraft levels under all flight conditions for both observer locations. Differences between the metrics generated via the CNoTE auralization and those generated by ANOPP are shown in parentheses. The differences are generally less than about $0.3 \mathrm{~dB}$, except for the sideline condition, where the differences are larger for both aircraft. The reader is again cautioned here not to add EPNL values for the three flight conditions for comparison with the cumulative certification values reported in the previous assessments, ${ }^{6}$ as the calculations were performed for different flight conditions.

It is useful to dissect the total aircraft EPNL to better understand where differences lie. For this purpose, comparisons of auralization generated EPNLs for isolated engine source components are shown in Figure 3 through Figure 9 for the centerline observer. As for the aircraft as a whole, the engine source components of the HWB have EPNL values lower than those of the SOA aircraft levels for all flight conditions. The reduction is primarily due to shielding. Absolute differences between EPNL generated via the CNoTE auralization and those generated from the ANOPP system noise prediction are shown as red bars. For the most part, differences are on the order of a few 
tenths of a dB. The largest differences are found in the tonal sources. The cause of these differences will be discussed in section IV.B. Note that comparisons of broadband airframe noise components are similar to the engine broadband components and are not shown for brevity. Similarly, comparisons at the sideline observer position are comparable and are not shown here. The favorable comparison of the CNoTE auralization and ANOPP integrated metrics demonstrates that the auralization methodology can handle the relevant component noise sources and be confidently applied to other flyover scenarios.

Table 5: Comparison of aircraft straight and level flyover EPNL (EPNdB).

Differences in predictions (ANOPP minus CNoTE) are also shown.

\begin{tabular}{|c|c|c|c|}
\cline { 2 - 4 } \multicolumn{1}{c|}{} & \multicolumn{3}{c|}{ Centerline observer (Difference) / Sideline observer (Difference) } \\
\cline { 2 - 4 } \multicolumn{1}{c|}{} & S\&L Approach & S\&L Sideline & S\&L Cutback \\
\hline \multirow{2}{*}{ SOA Aircraft } & $99.39(0.27) /$ & $101.69(0.70) /$ & $93.50(0.24) /$ \\
& $88.23(-0.09)$ & $97.90(-0.42)$ & $90.62(0.05)$ \\
\hline \multirow{2}{*}{ HWB C11 Aircraft } & $83.87(0.18) /$ & $90.60(0.36) /$ & $83.72(0.36) /$ \\
& $76.81(-0.07)$ & $88.77(0.46)$ & $83.02(0.19)$ \\
\hline
\end{tabular}

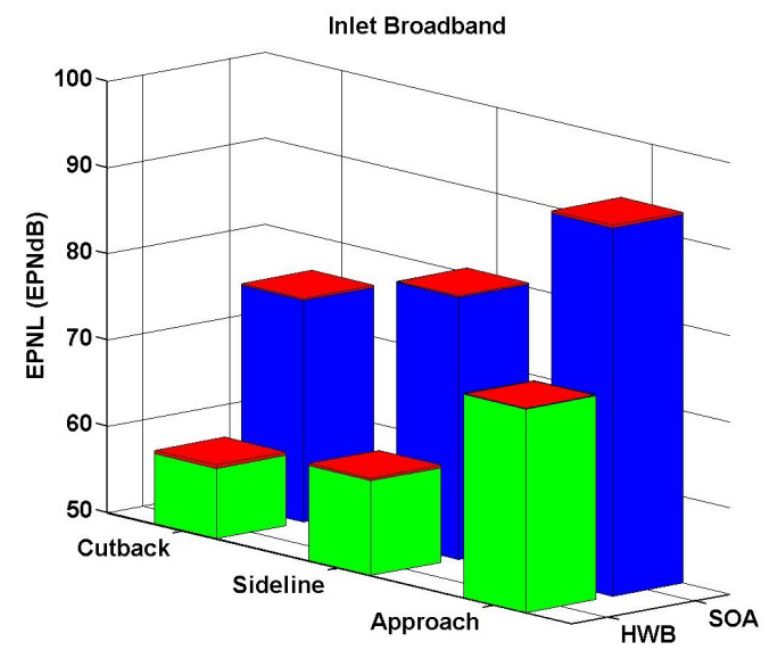

Figure 3: EPNL for fan inlet broadband noise for three S\&L flyover conditions.

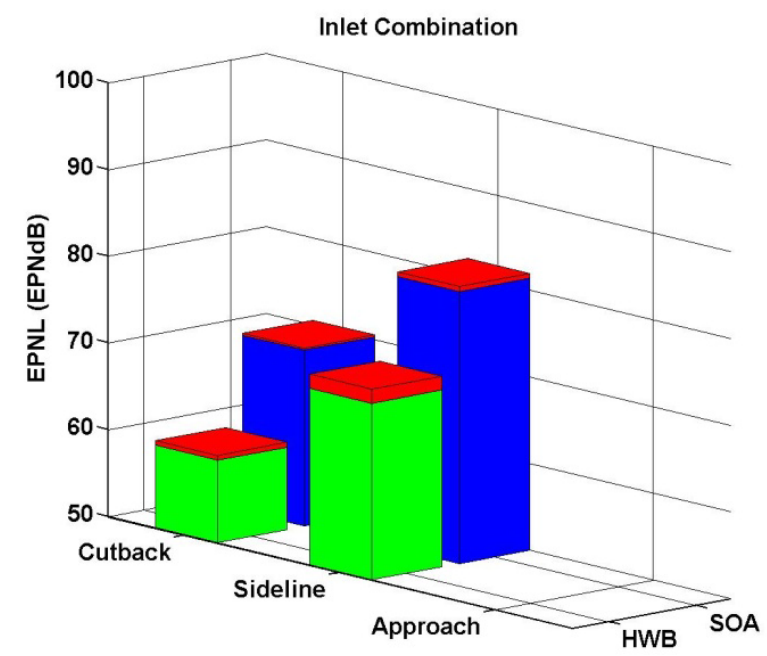

Figure 5: EPNL for fan inlet combination tonal noise for S\&L sideline and cutback flyover conditions.

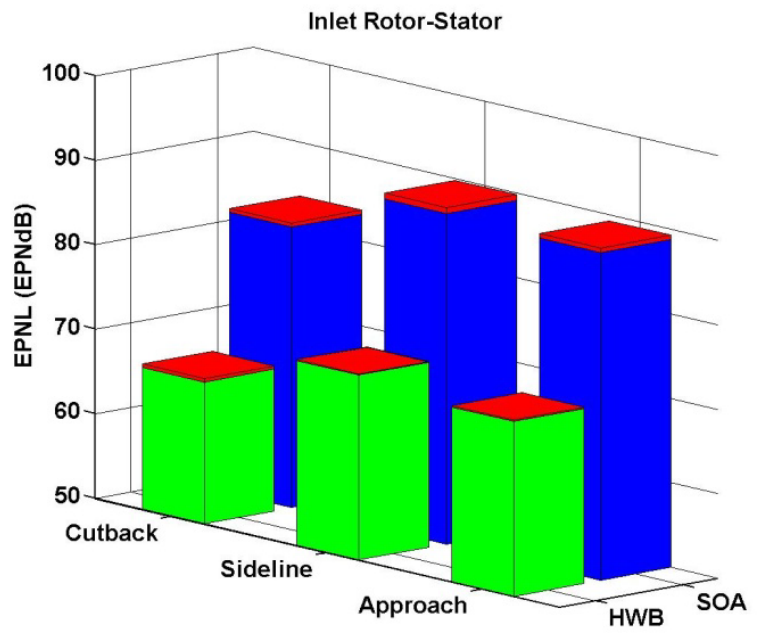

Figure 4: EPNL for fan inlet R-S tonal noise for three $S \& L$ flyover conditions.

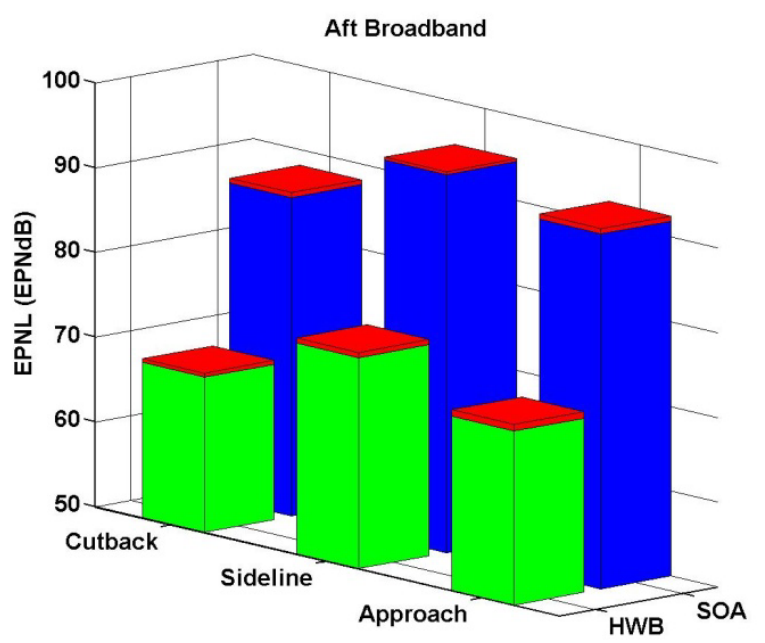

Figure 6: EPNL for fan aft broadband noise for three S\&L flyover conditions. 


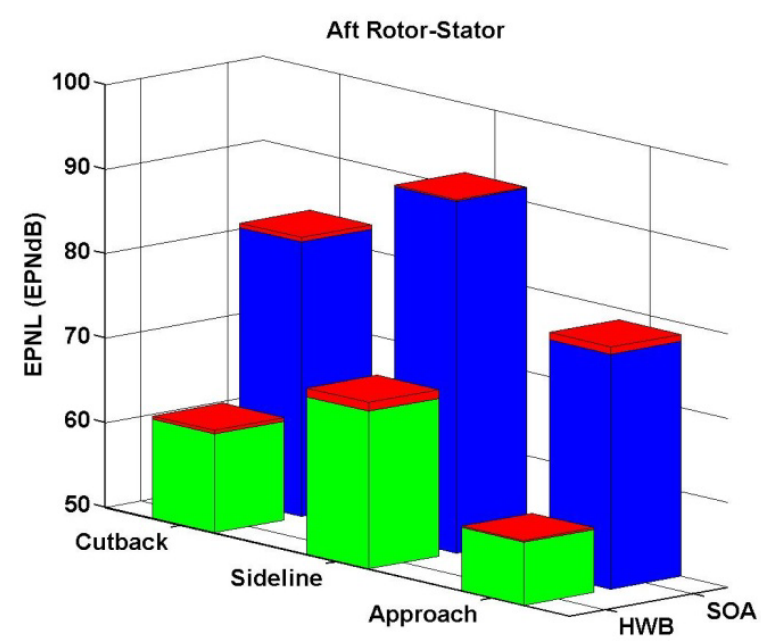

Figure 7: EPNL for fan aft R-S tonal noise for three S\&L flyover conditions.

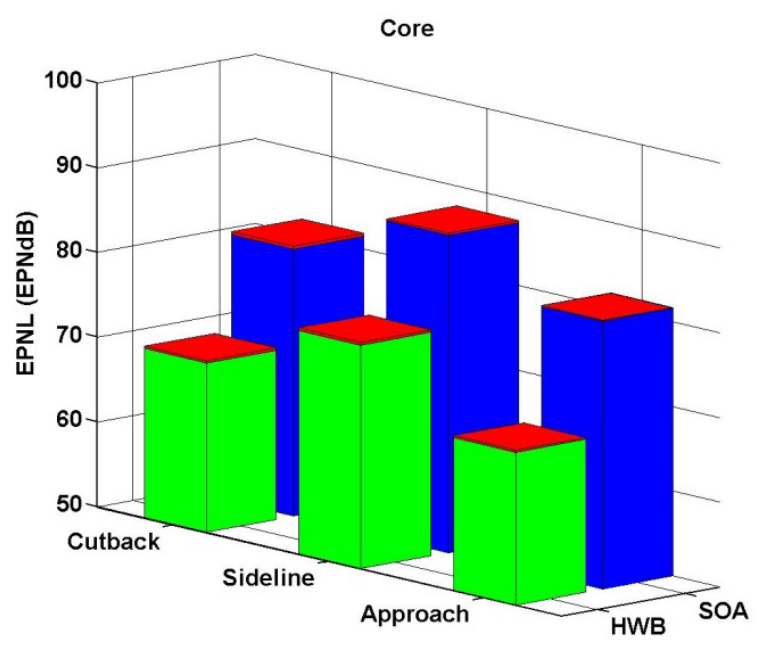

Figure 8: EPNL for core noise for three S\&L flyover conditions.

\section{B. Metric Time Histories}

The nature of differences between the CNoTE auralization and system noise predictions are better understood through inspection of time-varying metrics, in particular, the PNLT and A-weighted sound pressure level $\left(\mathrm{SPL}_{\mathrm{A}}\right)$. Consider the metric time histories shown in Figure 10 through Figure 12 for the three HWB C11 straight and level flyover conditions. Also shown in these figures are the time histories of the corresponding directivity angles and the nominal $10 \mathrm{~dB}$ down cut-off for the EPNL calculations. The differences in EPNL noted in Table 5 between the CNoTE auralization and the ANOPP can be largely attributed to the irregularity of the PNLT traces. This, in turn, is attributable to the tone correction as evidenced by the much smoother $\mathrm{SPL}_{\mathrm{A}}$ traces.

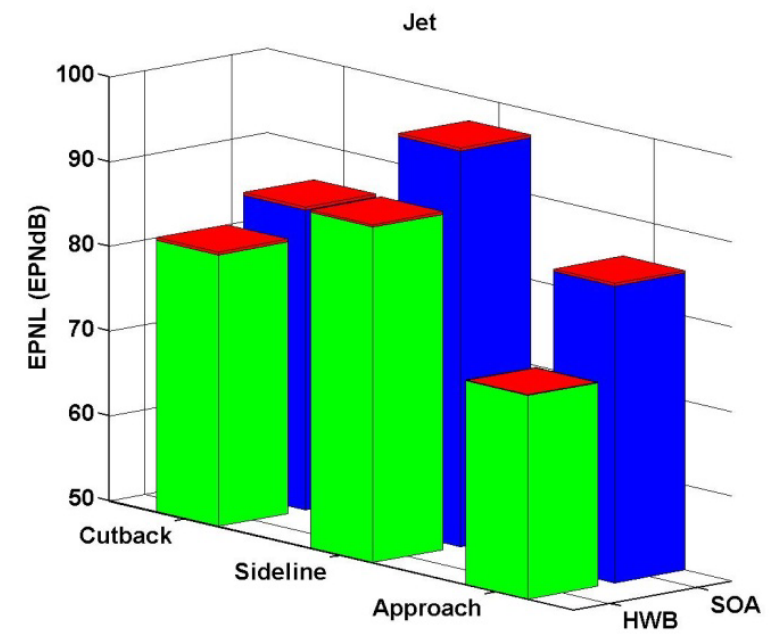

Figure 9: EPNL for jet noise for three S\&L flyover conditions.

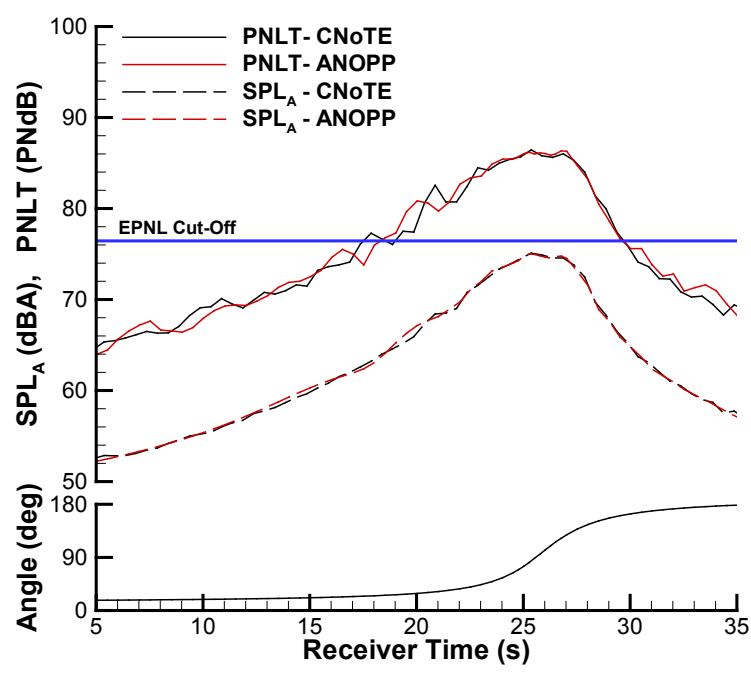

Figure 10: Aircraft PNLT and $\mathrm{SPL}_{\mathrm{A}}$ time histories for the HWB S\&L approach condition at the centerline observer. 


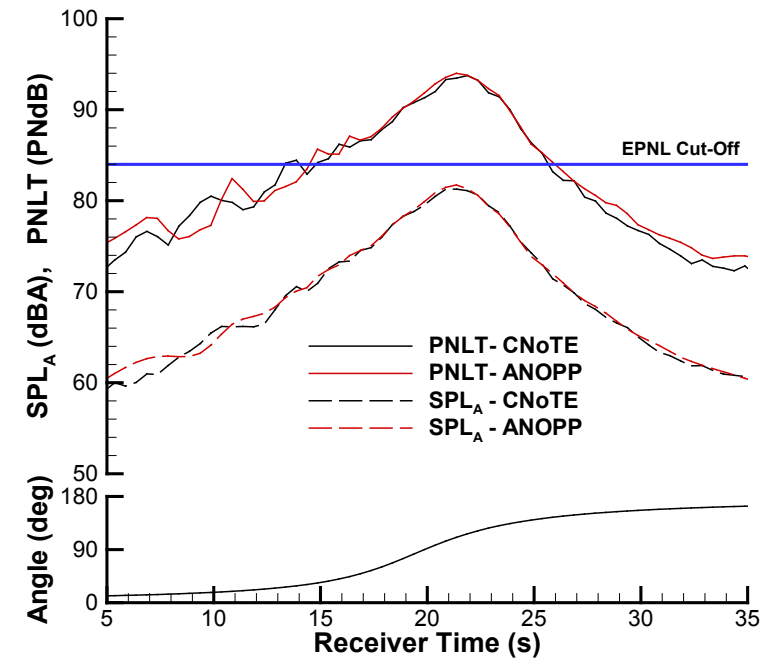

Figure 11: Aircraft PNLT and $\mathrm{SPL}_{\mathrm{A}}$ time histories for the HWB S\&L sideline condition at the centerline observer.

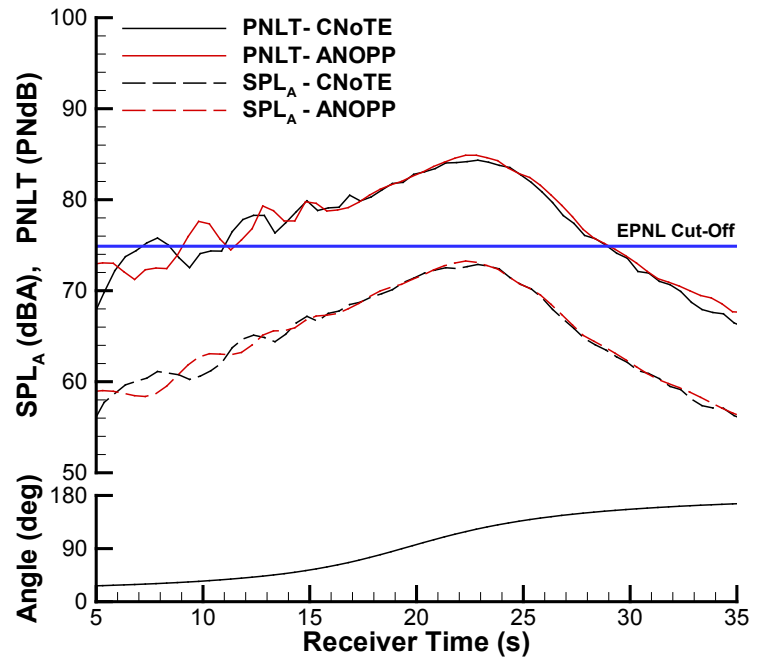

Figure 12: Aircraft PNLT and $\mathrm{SPL}_{\mathrm{A}}$ time histories for the HWB S\&L cutback condition at the centerline observer

It is worthwhile to consider how the tone correction varies between different source components. For this purpose, the PNLT and $\mathrm{SPL}_{\mathrm{A}}$ time histories for isolated jet and aft-radiated R-S interaction tones are shown in Figure 13 and Figure 14, respectively, for the HWB C11 straight and level sideline flight condition. The EPNL cutoff line shown in these figures correspond to the particular component. There is a great deal more irregularity indicated in the tone metric time histories than in the broadband jet metric time histories. Differences in the irregularity between the CNoTE auralization and ANOPP, not the irregularity itself, account for the larger component EPNL differences for tonal sources indicated in Figure 4, Figure 5, and Figure 7. Further, the differences are also noted in the $\mathrm{SPL}_{\mathrm{A}}$, indicating that they are not simply due to differences in the tone correction.

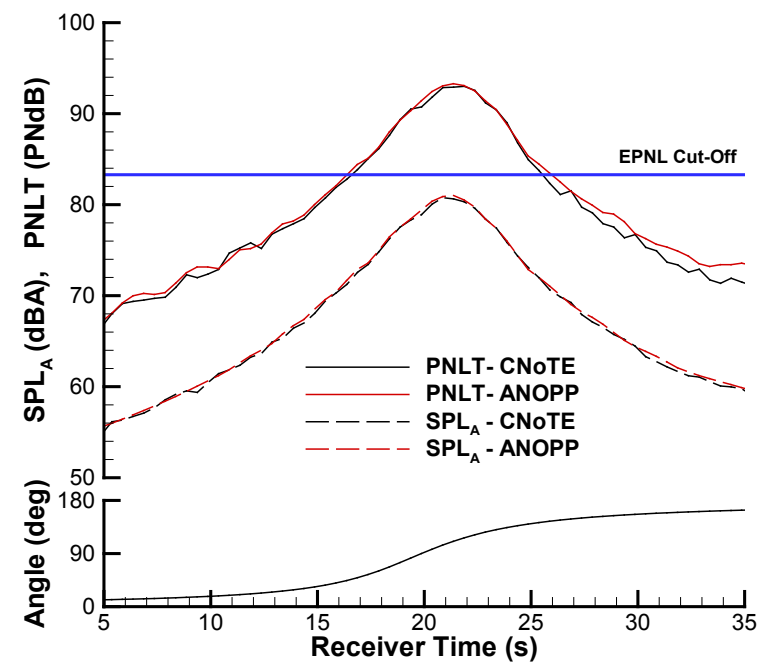

Figure 13: Jet noise PNLT and $\mathrm{SPL}_{\mathrm{A}}$ time histories for the HWB S\&L sideline condition at the centerline observer.

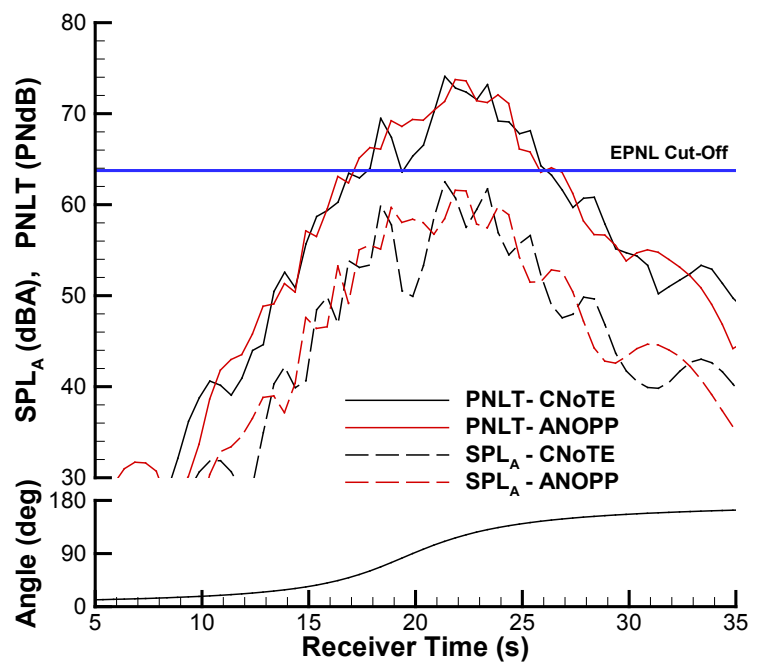

Figure 14: Aft R-S noise PNLT and $\mathrm{SPL}_{\mathrm{A}}$ time histories for the HWB S\&L sideline condition at the centerline observer.

The differences can be traced to the fact that the CNoTE auralization retains the phase relationship between the direct and ground plane reflected rays because propagation is performed in the time domain. While the direct and reflected spectra are time delayed in ANOPP, the proper phase relationship is lost because tones are lumped within $1 / 3$-octave bands. Note that the broadband jet noise is less sensitive to this phenomenon because the phase distribution is random. The above conclusion is substantiated by the corresponding metric time histories computed 
without the ground plane, as shown in Figure 15 and Figure 16. Here it is seen that the jet noise metric distribution is not significantly affected, while the tonal distribution is significantly smoother. While the presence of the hard ground plane increases the observer levels by about $3 \mathrm{~dB}$ over the no-ground case, it has a more striking affect on the auralized pseudo-recording, as is next demonstrated.

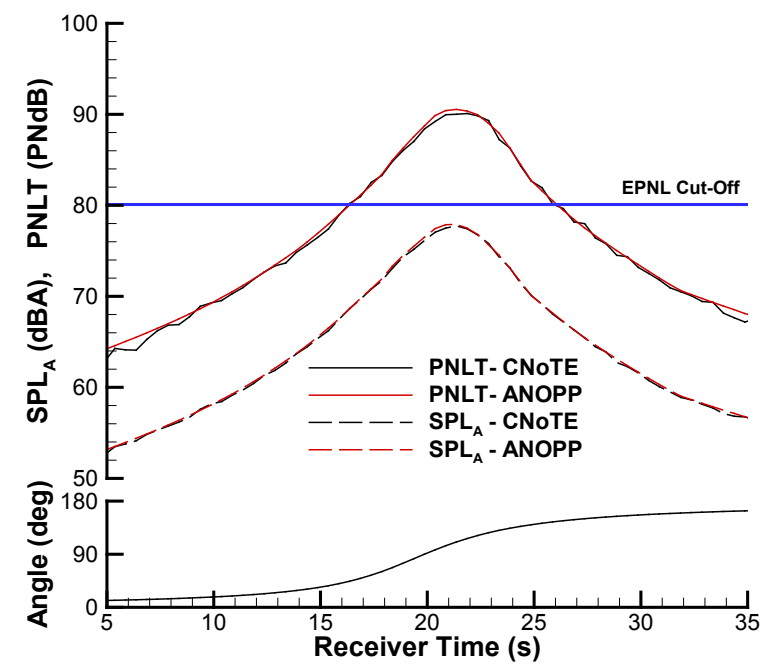

Figure 15: Jet noise PNLT and $\mathrm{SPL}_{\mathrm{A}}$ time histories for the HWB S\&L sideline condition at the centerline observer (ground plane removed).

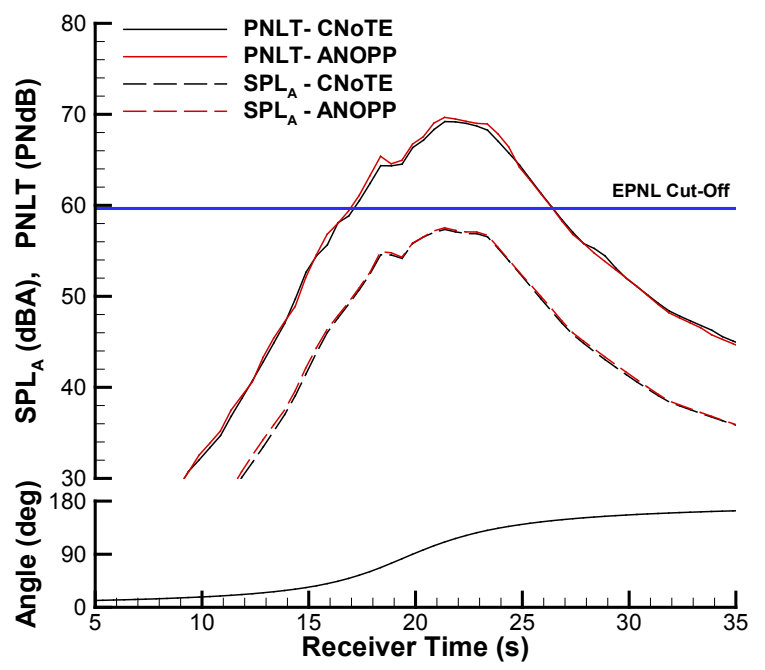

Figure 16: Aft R-S noise PNLT and $\mathrm{SPL}_{\mathrm{A}}$ time histories for the HWB S\&L sideline condition at the centerline observer (ground plane removed).

\section{Auralizations}

Spectrograms of the pseudo-recordings are shown in Figure 17 for each aircraft and straight and level flight condition at the centerline observer location. In each plot, the Doppler shift in the fan tones is evident and the interference created between the direct and ground plane reflected rays is prominent. The interference creates spectra notches which change with changes in the propagation path. The notches cut through both broadband and tonal noise. The effect of this interference on the PNLT time histories is most pronounced when the notches are widely separated, as seen at the beginning and end of each run in Figure 10 through Figure 12. As the notches are compressed near the overhead position, the tone correction is diminished and PNLT curve becomes smoother. The spectrogram of the HWB C11 straight and level sideline condition flyover without the ground plane is shown in Figure 18a. Absent is the interference pattern shown in Figure 17e. Consequently the tails of the component PNLT time histories shown in Figure 15 and Figure 16 are somewhat smoother than their ground reflected counterparts.

As the component EPNL figures suggest, the SOA flyover noise for the straight and level approach condition (a) is seen to be dominated by forward radiating components. The effect of shielding on the HWB C11 approach noise (b) is readily apparent as it significantly reduces the forward radiated broadband and tonal noise. The distinct twinpeak pattern as the aircraft passes overhead (between receiver times of about 23-28 seconds) is primarily due to the forward and aft radiated broadband fan noise. The effect of shielding on the HWB flyover noise is also evident in the straight and level sideline and cutback conditions where reduced broadband and tonal levels are also indicated. Recordings of each auralized flyover sounds are available for download via the Internet. ${ }^{32}$

\section{Effect of distributed sources}

As in the prior assessment work, ${ }^{6}$ all sources considered thus far have been collocated at the aircraft center of gravity. The effect of spatially distributed sources on the auralized sound was investigated by separating the two engines of the HWB C11 aircraft by a lateral distance of $28.7 \mathrm{ft}$. No attempt was made to account for differences in the shielding between port and starboard sides. The spectrogram of the CNoTE auralized sound at the sideline observer location for the sideline flight condition is shown in Figure 18b. The combination of the port and starboard side engine tones at slightly different Doppler shifted frequencies results in beating, which is seen as a tonal amplitude modulation separate from the interference pattern caused by the ground plane reflection. For this particular case, the effect is audible but not prominent. ${ }^{32}$ A negligible change in EPNL between collocated (88.77 EPNdB) and distributed sources $(88.72 \mathrm{EPNdB})$ is one reason why system noise assessments typically do not consider this effect. 


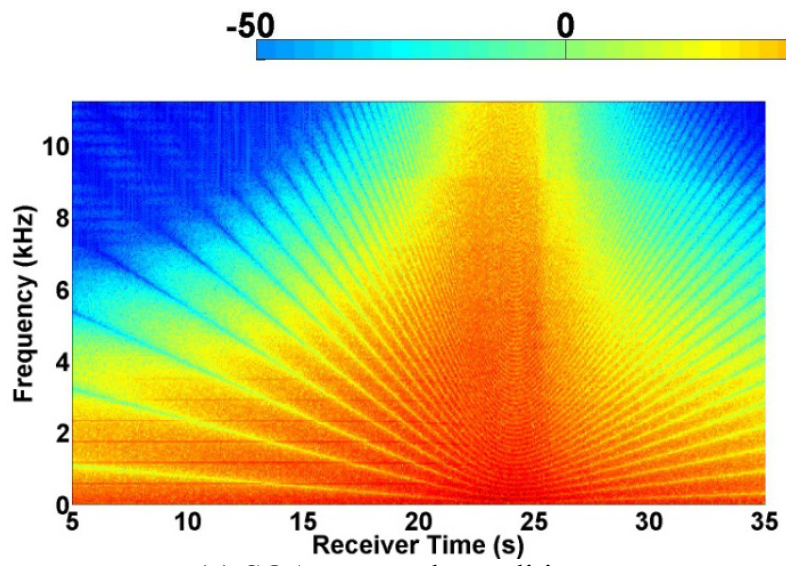

(a) SOA approach condition

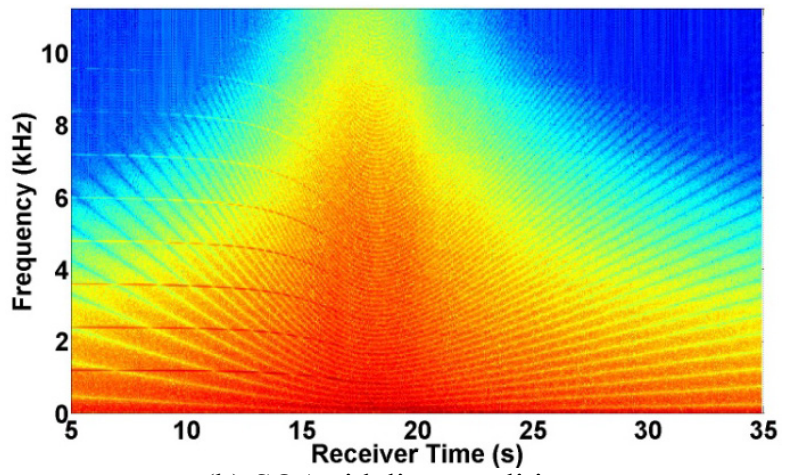

(b) SOA sideline condition

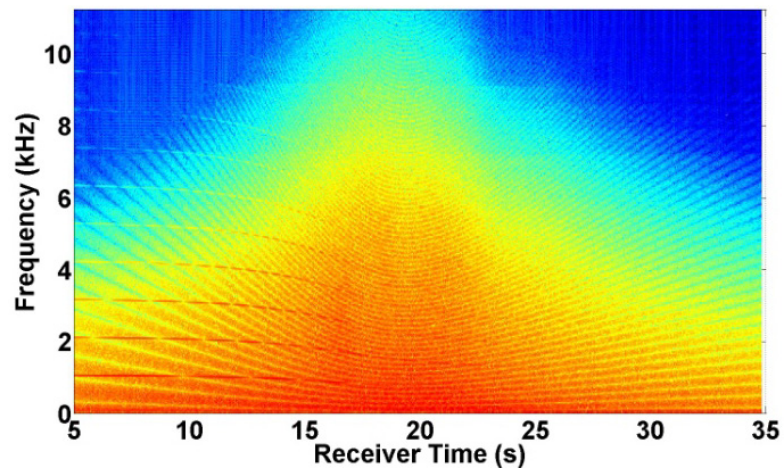

(c) SOA cutback condition
$50 \quad 100$ SPL (dB)

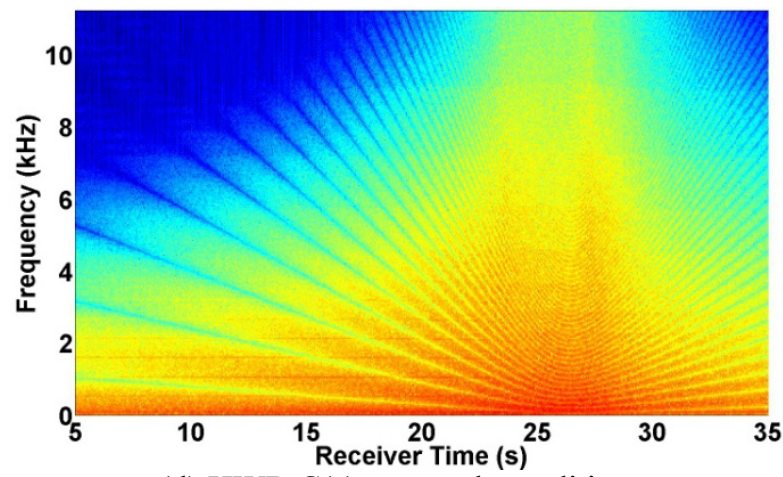

(d) HWB C11 approach condition

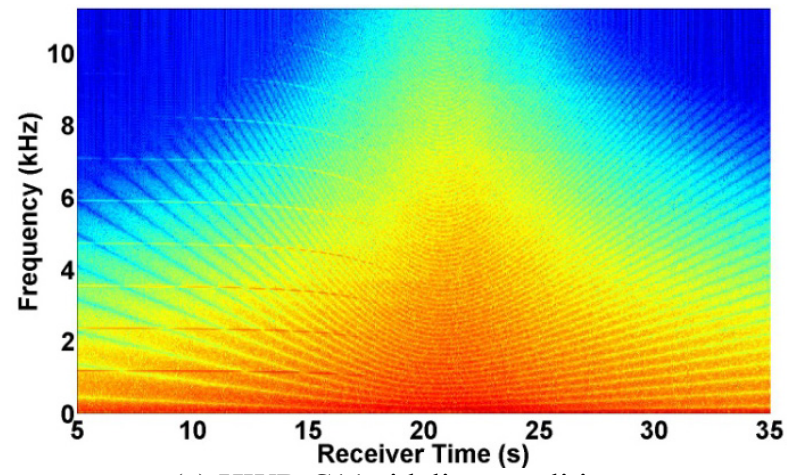

(e) HWB C11 sideline condition

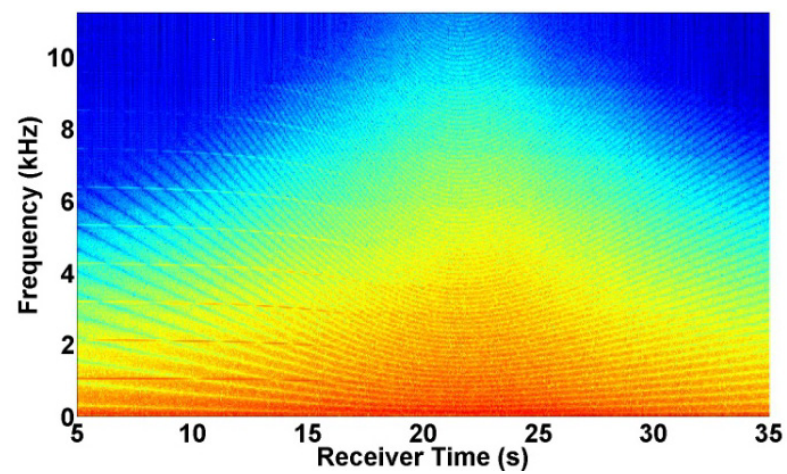

(f) HWB C11 cutback condition

Figure 17: Spectrogram of auralized flyover noise for SOA (a-c) and HWB C11 (d-f) aircraft in straight and level flight. [A large dynamic SPL range was used to aid visualization]

\section{Effect of low frequency oscillations (LFO)}

Low frequency oscillations were applied to the jet noise, R-S tones, and combination tones, as indicated in Table 2 , to determine that effect on the auralized sound. Shown in Figure 18c is the spectrogram of the auralized sound at the sideline observer location for the HWB C11 aircraft in the straight and level sideline flight condition. The amplitude modulation in the broadband jet noise is observed as a variation in time and frequency, and is audible in the pseudo-recording. ${ }^{32}$ Previous work ${ }^{21}$ indicated that variations in tonal fan noise were difficult to discern in the presence of broadband noise and this observation was affirmed in this particular case. ${ }^{32}$ A small difference in EPNL between the no LFO case (88.77 EPNdB) and the LFO case (88.42 EPNdB) was indicated. 
Finally, the pseudo-recording of the HWB C11 incorporating both LFO and distributed sources, represents the best obtainable auralization based on existing system noise predictions, shielding data, and empirical fluctuation data. As an example, the spectrogram for the sideline flight condition at the sideline observer location is shown in Figure 18d, with the audio clip available for download. Beating in the tones is less distinct than in Figure $18 \mathrm{~b}$ as the tone LFO modulates both amplitude and frequency.

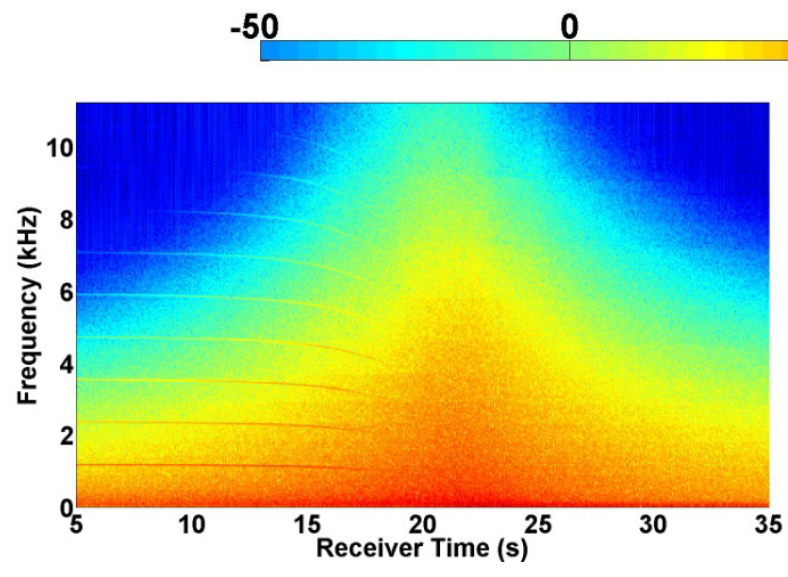

(a) Sideline flight condition without the effect of the ground plane (centerline observer)

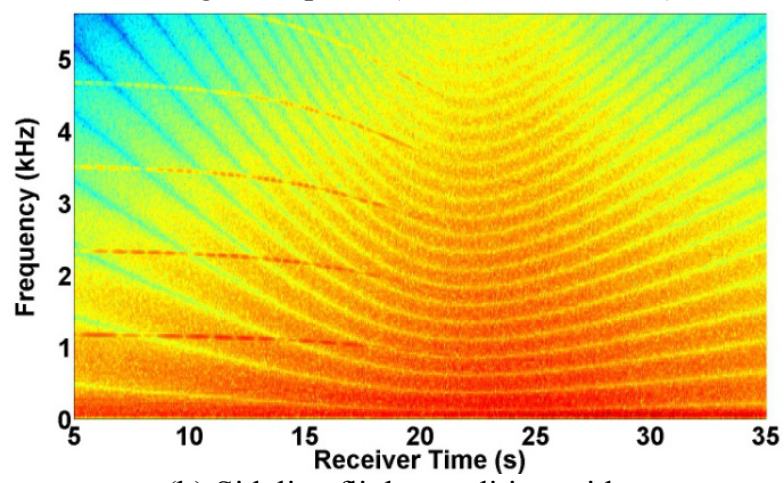

(b) Sideline flight condition with spatially distributed engines (sideline observer).
$50 \quad 100$ SPL (dB)

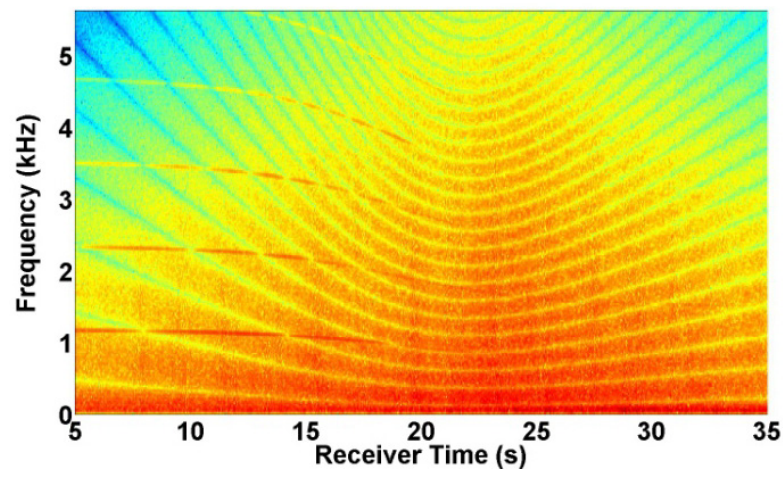

(c) Sideline flight condition with low frequency oscillations (sideline observer).

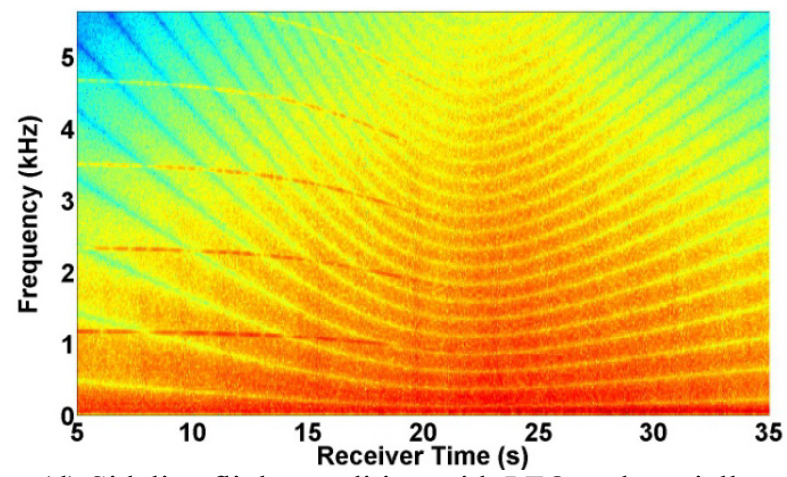

(d) Sideline flight condition with LFO and spatially distributed engines (sideline observer).

Figure 18: Spectrograms of auralized straight and level flyover noise for HWB C11 aircraft showing various simulation effects. [A large dynamic SPL range was used to aid visualization]

\section{Rendering in a Virtual Environment}

The flyover pseudo-recordings were cast within the CNoTE virtual environment using a binaural display. The captured audio and visual outputs at both observer locations are provided as movie clips downloadable from the Internet. ${ }^{32}$

\section{Conclusions}

Auralizations of a hybrid wing body and reference state-of-the-art tube-and-wing aircraft have been made possible using a combination of NASA system noise prediction tools (with experimentally determined shielding), and NASA synthesis and flyover simulation tools. These synthesis and simulation tools have been under development for several years, with the objective of producing flyover sounds that are both accurate, in terms of engineering quantities, and also realistic, in terms of perception by listeners. The effort reported herein represents a crucial step by coupling synthesis and simulation with the source noise prediction capabilities of the system noise prediction program ANOPP. Integrated metrics calculated from auralized flyover noise agree well with those obtained by ANOPP. Small differences in tonal source EPNL were found to be attributable to the manner in which the different methodologies propagate tones reflecting off a ground plane. The results demonstrate that auralization based upon source noise predictions is consistent with, and complementary to, system noise predictions alone. As a 
validated tool chain, auralization can be used with confidence to more effectively communicate the societal benefit of low noise concepts to stakeholders than can tabulated metrics alone. Further, auralization provides a feedback mechanism to the technologists developing noise reduction concepts. With this capability, it is now possible to assess human response to flyover noise by systematically evaluating source noise reductions within the context of a system level simulation.

While the present study considered only straight and level flight, additional work is needed to assess the benefit of HWB airframe shielding under other flight conditions, e.g. a banking turn which exposes some observer locations to unshielded or possibly amplified source noise radiated from the top of the vehicle. ${ }^{33}$ In addition, recent simulation developments allow curved propagation paths to be considered. ${ }^{23}$ This capability will permit the future study of upward radiated sound in atmospheres with downward refraction, e.g., in downwind conditions. Under such conditions, the benefits of HWB airframe shielding might also be reduced.

Additional development work is required to more closely integrate auralization and system noise prediction tools, in particular ANOPP2. ${ }^{28}$ As part of that activity, the compatibility of ground impedance models employed in the auralization and system noise prediction needs to be assessed since only a hard ground plane was considered in this work. The straight-line propagation used in the existing auralization framework needs to be changed to allow specification of an average speed of sound along the slant range for compatibility with the system noise prediction. This modification will allow non-uniform atmospheres to be considered in a compatible, albeit approximate manner. Finally, the manner in which source hemispheres are specified as a function of time needs to be reconciled to permit consistent simulation of arbitrary flight profiles under changing operating conditions. Only after these steps are taken can an auralization be made consistent with the prior assessments.

\section{Acknowledgments}

This work was performed with support from the Environmentally Responsible Aviation Project of the NASA Integrated Systems Research Program. The authors would like to acknowledge Mr. John Rawls, Jr., Northrop Grumman Corporation, Hampton, VA, for his significant contributions in the modification and running of ANOPP for this study and would like to thank Dr. Russell Thomas, NASA Langley Research Center, for inspiring this work.

\section{References}

1"National Aeronautics Research and Development Plan," National Science and Technology Council, Washington, DC, February 2010.

2"European Aeronautics: A Vision for 2020," Advisory Council for Aeronautics Research in Europe, January 2001.

${ }^{3}$ Collier, F.S., "Environmentally Responsible Aviation (ERA) Project," presentation at the Third NASA Fundamental Aeronautics Program Annual Meeting, Atlanta, Georgia, 2009.

${ }^{4}$ Czech, M.J., Thomas, R.H., and Elkoby, R., "Propulsion airframe aeroacoustic integration effects for a hybrid wing body aircraft configuration," International Journal of Aeroacoustics, Vol. 11, No. 3+4, 2012, pp. 335-368.

${ }^{5}$ Zorumski, W.E., "Aircraft noise prediction program theoretical manual," NASA TM-83199, 1982.

${ }^{6}$ Thomas, R.H., Burley, C.L., and Olson, E.D., "Hybrid wing body aircraft system noise assessment with propulsion airframe aeroacoustic experiments," International Journal of Aeroacoustics, Vol. 11, No. 3+4, 2012, pp. 369-410.

${ }^{7}$ Rizzi, S.A. and Sullivan, B.M., "Synthesis of virtual environments for aircraft community noise impact studies," 11th AIAA/CEAS Aeroacoustics Conference, AIAA-2005-2983, Monterey, CA, 2005.

${ }^{8}$ Rizzi, S.A., Sullivan, B.M., and Aumann, A.R., "Recent developments in aircraft flyover noise simulation at NASA Langley Research Center," NATO Research and Technology Agency AVT-158 "Environmental Noise Issues Associated with Gas Turbine Powered Military Vehicles" Specialists' Meeting, Paper 17, Montreal, Canada, 2008, NATO RTA Applied Vehicle Technology Panel.

${ }^{9}$ Rizzi, S.A., Aumann, A.R., Allen, M.P., Burdisso, R., and Faller II, K.J., "Simulation of rotary and fixed wing flyover noise for subjective assessments (Invited)," 161st Meeting of the Acoustical Society of America, Seattle, WA, 2011.

${ }^{10}$ Allen, M.P., Rizzi, S.A., Burdisso, R., and Okcu, S., "Analysis and synthesis of tonal aircraft noise sources," 18 th AIAA/CEAS Aeroacoustics Conference, AIAA-2012-2078, Colorado Springs, CO, 2012.

${ }^{11}$ Kontos, K.B., Kraft, R.E., and Gliebe, P.R., "Improved NASA-ANOPP noise prediction computer code for advanced subsonic propulsion systems. Volume 2: Fan suppression model development," NASA Contractor Report 202309, December 1996.

${ }^{12}$ Guo, Y., "An improved landing gear noise prediction scheme," The Boeing Company, NASA Contract NAS1-NNL04AA11B (Task NNL06AB63T), November 2006.

${ }^{13}$ Guo, Y., "Empirical prediction of aircraft flap side edge noise," The Boeing Company, NASA Contract NAS1-00086 (Task NNL04-AD34T), August 2006.

${ }^{14}$ Herkes, W., "Modular engine noise component prediction system (MCP) technical description and assessment document," The Boeing Company, NASA Contract NAS1-97040, August 2001. 
${ }^{15}$ Emmerling, J.J., Kazin, S.B., and Matta, R.K., "Core engine noise control program. Volume III, Supplement 1 - Prediction methods," FAA-RD-74-125, III-I (Available from DTIC as AD A030 376), March 1976.

${ }^{16}$ Stone, J.R., Kresja, E.A., and Clark, B.K., "Jet noise modeling for suppressed and unsuppressed aircraft in simulated flight," NASA TM-2009-215524, March 2009.

${ }^{17}$ Sen, R., Hardy, B., Yamamoto, K., Guo, Y., and Miller, G., " Airframe noise sub-component definition and model," NASA CR2004-213255, September 2004.

${ }^{18}$ Fink, M., "Noise component method for airframe noise," AIAA Journal of Aircraft, Vol. 16, No. 10, 1979 , pp. $659-665$.

${ }^{19}$ Fink, M., "Airframe noise prediction method " U.S. Department of Transportation, Federal Aviation Administration, FAA-RD 77-29, 1977.

${ }^{20}$ Berton, J.J., Envia, E., and Burley, C.L., "An analytical assessment of NASA's N + 1 Subsonic Fixed Wing Project noise goal," 15th AIAA/CEAS Aeroacoustics Conference, AIAA-2009-3144, Miami, FL, 2009.

${ }^{21}$ Okcu, S., Allen, M.P., and Rizzi, S.A., "Psychoacoustic assessment of a new aircraft engine fan noise synthesis method," 164th Meeting of the Acoustical Society of America, Kansas City, MO, 2012.

${ }^{22}$ Grosveld, F.W., Sullivan, B.M., and Rizzi, S.A., "Temporal characterization of aircraft noise sources," Proceedings of the 42nd AIAA Aerospace Sciences Meeting, AIAA-2004-1029, Reno, NV, 2004.

${ }^{23}$ Arntzen, M., Rizzi, S.A., Visser, H.G., and Simons, D.G., "A framework for simulation of aircraft flyover noise through a nonstandard atmosphere," 18th AIAA/CEAS Aeroacoustics Conference, AIAA 2012-2079, Colorado Springs, CO, 2012.

${ }^{24}$ Laakso, T.I., Välimäki, V., Karjalainen, M., and Laine, U.K., "Splitting the Unit Delay -Tools for Fractional Delay Filter Design," IEEE Signal Processing Magazine, Vol. 13, No. 1996, pp. 30-60.

${ }^{25}$ "GoldServe, AuSIM3D Gold Series Audio Localizing Server System, User's Guide and Reference, Rev. 1d," AuSIM Inc. Mountain View, CA, October 2001.

${ }^{26}$ Delany, M.E. and Bazley, E.N., "Acoustical properties of fibrous absorbent materials," Applied Acoustics, Vol. 3, No. 2, April 1970, pp. 105-116.

${ }^{27}$ Zölzer, U., ed. DAFX - Digital audio effects. John Wiley \& Sons, Ltd., West Sussex, England, 2002.

${ }^{28}$ Lopes, L.V. and Burley, C.L., "Design of the next generation aircraft noise prediction program: ANOPP2," 17th AIAA/CEAS Aeroacoustics Conference, AIAA 2011-2854, Portland, Oregon, 2011.

${ }^{29}$ Begault, D.R., 3-D sound for virtual reality and multimedia. Academic Press, Inc., Chestnut Hill, MA, 1994.

${ }^{30}$ Pulkki, V., "Virtual Sound Source Positioning Using Vector-Base Amplitude Panning," Journal of the Audio Engineering Society, Vol. 45, No. 6, 1997, pp. 456-466.

31 "American National Standard Method for the calculation of the absorption of sound by the atmosphere," American Institute of Physics, ANSI S1.26-1995 (ASA 113-1995), 1995.

32" Aircraft flyover simulation," http://stabserv.larc.nasa.gov/flyover/, NASA, 2013.

${ }^{33}$ Tinetti, A.F. and Dunn, M.H., "Scattering of simulated broadband noise by conventional and next generation aircraft," $18 \mathrm{th}$ AIAA/CEAS Aeroacoustics Conference, AIAA-2012-2075, Colorado Springs, CO, 2012. 